\title{
Pain and mortality in older adults: The influence of pain phenotype
}

\author{
Diane Smith PhD ${ }^{1}$, Ross Wilkie PhD ${ }^{1}$, Peter Croft MD ${ }^{1}$, John McBeth PhD ${ }^{2}$
}

1. Arthritis Research UK Primary Care Centre, Research Institute for Primary Care \& Health Sciences, Keele University,

Staffordshire

United Kingdom

2. Arthritis Research UK Centre for Epidemiology, Centre for Musculoskeletal Research, Institute of Inflammation and Repair Faculty of Medical and Human Sciences, Manchester Academic Health Sciences Centre,

The University of Manchester, United Kingdom

Running head: Pain phenotype and mortality

Word count: 3764

All correspondence relating to the paper should be addressed to Diane Smith diane.smith@mmu.ac.uk

The authors' work was supported by Arthritis Research UK Primary Care Centre, Primary Care Sciences, Keele University. 


\begin{abstract}
Objective

Moderate to severe chronic pain affects one in five adults. Pain may increase the risk of mortality but the relationship is unclear. This study investigated whether mortality risk was influenced by pain phenotype, characterised by pain extent or pain impact on daily life.
\end{abstract}

\title{
Methods
}

The study population was drawn from two large population cohorts of adults aged $\geq 50$ years; the English Longitudinal Study of Ageing (ELSA) $(n=6324)$ and the North Staffordshire Osteoarthritis Project (NorStOP) ( $n=10985)$. Survival analyses (Cox's proportional hazard models) estimated the risk of mortality in participants reporting "any pain" and then separately according to the extent of pain (total number of pain sites; widespread pain according to American College of Rheumatology (ACR) criteria; widespread pain according to Manchester criteria) and pain impact on daily life (pain interference; and often troubled with pain). Models were cumulatively adjusted for age, sex, education and wealth/adequacy of income.

\section{Results}

After adjustments, the report of any pain (MRR 1.06, 95\% $\mathrm{Cl}(0.95,1.19)$ ) or having widespread pain $(A C R 1.07(0.92,1.23)$ or Manchester $1.16(0.99,1.36))$ was not associated with an increased risk of mortality. Participants who were often troubled with pain (1.29 $(1.12,1.49))$ and those that reported "quite a bit" $(1.38(1.20,1.59))$ and "extreme" $(1.88$ $(1.54,2.29))$ pain interference had an increased risk of all-cause mortality. 


\section{Conclusion}

Pain that interferes with daily life, rather than pain per se was associated with an increased risk of mortality. Future studies should investigate the mechanisms through which pain increases mortality risk. 


\section{Significance and Innovations}

- Pain impact is key to understanding the relationship between pain and mortality.

- An investigation of potential mechanisms of a relationship between pain and mortality is warranted. 
Pain has a major impact on individuals and society (1), is acknowledged by the World Health Organisation and the United Nations as a global problem, and is increasingly recognised as a condition in its own right (2). A significant proportion of people in the general population develop persistent disabling pain, and this contributes to the high number of years lost to disability attributed to painful conditions (3). However, pain may also carry an increased risk of mortality, although the risk is unclear and the evidence contradictory $(4,5)$. Some of the risk may be attributed to underlying disease such as cancer, which is considered to cause the pain [3], but the important question of whether the experience or nature of chronic pain confers a risk of premature death is unresolved $(6,4,7,8,5,9-13)$.

A recent systematic review and meta-analysis of the relationship between chronic pain and mortality (14) indicated a modest but non-significant risk of mortality for people with pain, but with high heterogeneity between studies. An important source of variability was differences in pain phenotype between studies, with heterogeneous definitions of pain defined on the basis of pain extent (number of locations), duration and impact. Previous studies have suggested that mortality risk may be influenced by pain extent and duration $(8,15)$. For example, strong associations have been reported between chronic widespread pain and mortality (MRR 2.8; 95\% Cl 1.3,6.1) (9); and people with fibromyalgia who satisfied the more stringent American College of Rheumatology (ACR) 2010 criteria had an increased risk of mortality when compared to those satisfying the less stringent 1990 criteria only (HR $1.62 ; 95 \% \mathrm{Cl} 1.19,2.21)(11,16)$.

The aim of this study was to investigate the effect of pain phenotype on the association between pain and mortality in older adults, using two different population cohorts, and to 
test the hypothesis that the association with mortality increases with increasing pain extent and impact on daily life.

\section{Materials and Methods}

\section{Design Overview, Setting and participants}

Two population based cohort studies of older adults were used in this study: the English Longitudinal Study of Ageing (ELSA) (17) and the North Staffordshire Osteoarthritis Project (NorStOP). The data collection methods for these studies are described in detail elsewhere $(18,19)$. Briefly, ELSA is a large scale longitudinal panel study of the health, economic and social circumstances of adults aged 50 years and over in England which began in 2002. NorStOP is a cohort study designed to assess the prevalence and impact of pain in a community sample of adults aged 50 years and over in North Staffordshire, England. NorStOP also began in 2002. Pain and putative confounders were identified using data from wave 2 (collected in 2004) of ELSA and the NorStOP baseline samples (collected during 2002 to 2005). A total of 6324 (67\%) of participants in the ELSA and 10985 (59\%) of the NorStOP study participants had complete predictor, outcome, and putative confounders data (including characteristics tested as mediators in a subsequent study) and were used in this analysis. (Figures $\mathrm{i}$ and ii in the supplementary material display how these samples were derived).

Pain phenotypes (see Table i in supplementary material for details)

\section{Often troubled}

Participants in the ELSA were asked whether they were often troubled with pain. Those replying "yes" were classified as being often troubled with pain. 


\section{Severity}

Participants who were classified as being often troubled with pain were then asked to rate the severity of their pain as mild, moderate, or severe.

\section{Any pain}

Participants in the NorStOP study were asked "In the past 4 weeks have you had pain that has lasted for one day or longer in any part of your body (not including pain from illnesses such as flu)?" Those participants who responded "Yes" were categorised as having "any pain".

\section{Widespread pain (ACR criteria)}

Participants in the NorStOP study classified as having "any pain" were then asked to shade the site of their pain on a blank body manikin (front and back views). Based on their pain reports those satisfying the ACR criteria for widespread pain (pain in the axial skeleton, on the right and left sides of the body and above and below the waist) (20) were identified.

\section{Widespread pain (Manchester criteria)}

Participants in the NorStOP study who satisfied the Manchester criteria for widespread pain (pain in both the axial skeleton and at least two sections of two contralateral limbs) (21) were also identified.

\section{Number of pain sites}

In addition, for participants in the NorStOP study the number of shaded areas on the body manikin (one to 44) was calculated and categorised into quartiles of 1-3 sites, 4-6 sites, 7-11 sites and $12+$ sites of pain. 


\section{Pain interference}

Participants in the NorStOP study were asked to complete the Medical Outcomes Study (MOS) Short Form (SF)-12 (22) . The MOS SF12 includes the question "During the past 4 weeks how much did pain interfere with your normal work (including both work outside the home and housework)?” ('Extremely', 'Quite a bit', 'Moderately', 'A little bit', 'Not at all').

\section{Vital status}

Vital status for the ELSA sample was obtained from the Office of National Statistics (ONS) and for the NorStOP sample from the patient registration system held at the local Primary Care Trust and from dates of death notified to the NorStOP project team. These latter dates of death were confirmed using manual tracing of the NHS Summary Care Record Demographic system.

The statistical analysis used Cox's proportional hazard modelling to assess the risk of death according to pain phenotype (see below for details). In order to calculate hazard ratios, it was necessary to determine the length of time until the event (death) or censoring took place.

For participants in the ELSA, information on year of death was available until the end of February 2012; time in the study was calculated in months from the date of interview until the $31^{\text {st }}$ December in the year the participant was known to have died (or $28^{\text {th }}$ February if they died in 2012). The censor date for those who survived was the $28^{\text {th }}$ February 2012. 
For participants in the NorStOP study, the number of days participants remained in the study from the date of questionnaire completion to the date of death or censoring was used. Information on vital status was available until $1^{\text {st }}$ October 2012.

\section{Putative confounders}

Demographic variables were age, sex and socio-economic status indicated by educational attainment and current wealth (ELSA)/adequacy of income (NorStOP). These were considered to be the major potential confounders of the mortality-phenotype link according to the analytic strategy used in this study. In both datasets age was categorised into year bands $50-59,60-69,70-79$ and 80 and over.

For educational attainment, in ELSA participants were asked to look at a card listing educational qualifications and indicate which, if any, they held; participants were categorised to the highest qualification they held (National Vocational Qualification (NVQ)4/5 or degree or equivalent, higher education (university) below degree, NVQ3/Advanced level equivalent, NVQ2/Ordinary level equivalent, NVQ1/Certificate of Secondary (high school) education (CSE) or equivalent or foreign/other or no qualification). In NorStOP educational attainment was assessed by asking participants to indicate whether they had continued full-time education beyond school (response options 'yes/no') which formed a dichotomous variable indicating school education/further education.

For wealth, in ELSA net total non-pension wealth, was categorised by using quintiles to provide five groups labelled from low to high. This measure of wealth has been found to be the strongest socioeconomic predictor of health in the ELSA sample (23). In NorStOP, perceived adequacy of income was measured with a single item. Participants were asked to 
compare the adequacy of their income with their living costs and indicate their situation (find it a strain to get by from week to week/ have to be careful with money/ able to manage without much difficulty/ quite comfortably off). This item measures a range of financial circumstances (from affluence to poverty) without eliciting details of responders' income and is robust indicator of financial capacity in older adults (24).

\section{Statistical analysis}

A comparison between the study samples (ELSA $n=6324$, NorStOP 10985) and larger samples containing participants with only predictor, outcome and putative confounder information (ELSA $n=8572$, NorStOP $n=14023$ ) i.e. those without complete data for the characteristics tested as mediators in a subsequent study (see figures $\mathrm{i}$ and ii in supplementary data for details) was undertaken to assess for potential selection bias. A weighted analysis (where weights were calculated using age, sex, education and wealth/income variables and applied to the study sample to estimate the effects in the larger sample) was also undertaken to assess for selection bias. The distribution of demographic and socioeconomic factors was examined by vital status in both samples.

Cox's proportional hazard modelling was used to assess the risk of death for each pain phenotype, using time to death as the outcome. First, univariate models were constructed to examine the association between each pain phenotype and mortality (Model 1). Then in multivariable models the association for each phenotype was cumulatively adjusted for age and sex (Model 2) and then education and wealth (ELSA)/adequacy of income (NorStOP) (Model 3). The assumption of proportionality for each of the survival models was tested using Schoenfeld tests and plots of Schoenfeld residuals. These assumptions were valid for "often troubled" with pain, pain severity, any pain, widespread pain (ACR and widespread 
pain (Manchester criteria). For pain interference, Schoenfeld tests indicated the assumption of proportionality did not hold, and so the period was divided into two periods in accordance with previous research (25); the first period ranged from 0-365 days (0-1 year) and the second period was $>366$ days (i.e. 2-10 years). The proportionality assumption was met for both of these periods.

Stata version 13 was used for all analyses. The results of the Cox's proportional hazard models are presented as Mortality Rate Ratios (MRRs) with 95\% confidence intervals (CI).

\section{Results}

\section{Sample characteristics}

\section{ELSA}

There were 6324 participants included in the ELSA sample for the analysis of the relationship between mortality and often troubled with pain and pain severity. The median age of the sample was 63 years and $55 \%$ were female (Table ii supplementary data). Those with missing data were more likely to be troubled with pain, have greater severity of pain, be older, female, have lower levels of education and be financially poorer than those with complete data (Table ii supplementary data).

The length of time in the study for those who died $(n=764(12.1 \%))$ ranged from 0 months to 90 months from the date of interview, with a mean survival time of 54.1 months (SD 24.7). Within 12 months of the interview date forty deaths (5.2\%) occurred. The proportion of the sample reported being "often troubled" with pain was $35.9 \%$ with $6.5 \%$ reporting this to be of severe intensity (Table iii supplementary data). The proportion of the sample educated to degree level or above was 14.5\%. Participants who died during 8 years of follow-up ( $n=764)$, 
compared with those who remained alive $(n=5560)$, were older, and more likely to be male, lower socioeconomic status (i.e. lower education and wealth), often troubled with pain, and reported greater severity of pain (Table iii supplementary data).

\section{NorStOP}

There were 10985 participants included in the NorStOP sample for the analysis of the relationship between mortality and any pain, widespread pain (ACR and Manchester criteria), number of pain sites and pain interference. The median age was 63 years and $54 \%$ of the sample were female.

Participants with complete data were more likely to be younger, less likely to be female, be better educated, have greater adequacy of income, and report pain interference than those with missing data (Table iv supplementary data).

The length of time in the study for participants who died $(n=1484(13.5 \%))$ ranged from 7 days to 3823 days from the date of response with a mean survival time of 2036 days (SD 1052). Within 12 months from the date of response $6.5 \%(n=97)$ of deaths occurred. The proportion of the sample reporting "any pain" was $71.2 \%$, with $25.0 \%$ of the sample meeting the ACR criteria for widespread pain and $15.7 \%$ meeting the Manchester criteria for widespread pain (Table iv supplementary data). The proportion of the sample reporting pain at 12 anatomical sites or more was $18.8 \%$ and $5.5 \%$ of the sample reported extreme pain interference. Participants who died in the NorStOP sample were more likely to be older, male, of lower socioeconomic status (measured according to education and income), and more likely to report pain interference. There were no differences in the report of any pain, 
ACR WP, Manchester WP, and number of pain sites between those who died and those who remained alive (Table $v$ supplementary data).

An exploration of the relationship between the number of pain sites and pain interference variable used in this study was undertaken (Table 1). The highest proportion of deaths occurred for those reporting only 1-3 sites of pain but extreme pain interference (37.5\%).

The relationship between pain phenotypes and mortality

\section{Often troubled with pain (ELSA cohort)}

The rate of mortality was higher in those often troubled with pain when compared to those not often troubled with pain (Figure iii supplementary data) and this remained significant when adjusted putative confounders (Table 2).

\section{Pain severity (ELSA cohort)}

When compared to those reporting they were not "often troubled" with pain, participants who reported being often troubled with mild pain were no more likely to die over the follow up period, but participants reporting being often troubled by moderate or severe pain had increased mortality risks. These latter two estimates attenuated but remained significant when adjusted for age and sex (Model 2) and for age, sex, education and wealth (Model 3). The weighted analysis had little effect on the results (Table 2).

\section{Any pain (NorStOP cohort)}

Visually, the mortality rate was higher across the study period for those with "any pain" compared to those with no pain (Figure iv supplementary data). However, there was no 
statistically significant increased risk of mortality for participants with "any pain" (Model 1) with no change when adjusted for potential confounders (Models 2 and 3) (Table 3).

\section{Widespread pain (ACR criteria) (NorStOP cohort)}

There was no association between mortality and widespread pain by the ACR criteria or for widespread pain that did not meet the ACR criteria (Table 3).

\section{Widespread pain (Manchester criteria) (NorStOP cohort)}

For participants with widespread pain that met the Manchester criteria there was an increased risk of mortality in the analyses adjusted for age and sex only. There was no increased risk of mortality for pain that did not meet the Manchester criteria for widespread pain (Table 3).

\section{Number of pain sites (NorStOP cohort)}

There was a significant increased risk of mortality for participants with 12 or more pain sites adjusted for age and sex only. There was no trend of increased mortality risk with number of pain sites (Table 3).

The weighted analysis for all of the above phenotypes had little effect (Table 3).

\section{Pain interference (NorStOP cohort)}

Pain interference was associated with increased mortality over time (Figure v supplementary data). The risk of mortality associated with pain interference was higher in the first year of follow up than for the following 9 years (Tables $4 \& 5$ ). There were 97 deaths in the first 12 months, with risk associated with increasing pain interference. " $\mathrm{A}$ little" and "moderate" pain interference were not associated with increased mortality; pain 
that interfered "quite a bit" was associated in the crude model and when adjusted for age and sex, but this attenuated and became non-significant when adjusted for age, sex, education and adequacy of income. "Extreme" pain interference was associated with an increased risk of mortality in all models (Table 4).

In the period 366 days to 10 years of follow up, there was no increased risk of mortality for participants with "a little" pain interference in the crude or adjusted models. For participants who indicated "moderate" pain interference there was an increased risk of mortality in the crude model only which attenuated and became non-significant when adjusted for confounders. Compared to those with no pain, participants reporting "quite a bit" or "extreme" pain interference had an increased risk of mortality in all models (Table 5). A weighted analysis was not repeated for the pain interference phenotype due to the need to split the analysis into time bands but from the previous results it is assumed this would have little effect.

\section{Discussion}

\section{Summary of findings}

This study investigated the association between pain phenotype and mortality in two large population-based datasets. For the first time, we found the strongest association with mortality was a measure of the impact or troublesomeness of pain in daily life, consistent across both cohorts studied, whilst the presence of pain (yes/no) was not associated.

\section{Pain impact}

The magnitude of the relationship observed in the ELSA dataset between "troubling" pain and all-cause mortality (MRR 1.29; $95 \% \mathrm{Cl} 1.12,1.49$ ) was consistent with the association 
between pain that interferes with daily life (to a moderate extent or more) and mortality in the NorStOP study. This consistency between the two cohorts adds to the credibility of this finding.

By contrast with some previous findings from the literature, measures of the presence and extent of pain were not consistently associated with mortality risk. The phenotypes of pain extent tested in the NorStOP sample were comparable to phenotypes used in other studies $(4,7)$, but the results did not confirm those studies. Macfarlane et al., 2001 used the ACR criteria to categorise widespread pain and found a $30 \%$ increased risk of mortality among those meeting the criteria after adjusting for age, sex and study location (4), confirmed in a later study by McBeth et al., (2009) that additionally adjusted for ethnic group and Townsend score of deprivation (7). However, in the current study, participants meeting the ACR criteria for widespread pain did not have an increased risk of mortality.

The findings from the NorStOP sample regarding the number of pain sites were unexpected as associations between increasing number of pain sites and a reduction in overall health, sleep quality, psychological health and decreased function (26) and increased mortality (7) have been reported. In the current study, the highest proportion of deaths occurred for those reporting only 1-3 sites of pain but extreme pain interference (37.5\%) (Table 1). Although there were only a small number of participants in this category, the finding does suggest that the link is with impact rather than with pain and pain extent per se. There is some correlation between a greater number of pain sites and the extent of pain interference (Table 1), but in the multivariable models it is pain interference, and not number of pain sites, that is independently associated with increased mortality in this study. 
We conclude that the presence and extent of pain is not related to mortality but the impact that pain has on an individual's daily life is.

\section{Strengths and limitations}

Pain prevalence

Although there were some contrasts in the findings from the phenotypes in the NorStOP sample compared to previous studies, the pain phenotype data in NorStOP was collected using a validated approach and found in previous analyses to be reliable (27). Our prevalence estimates for pain and widespread pain in the NorStoP sample are higher than those reported in previous UK population-based studies such as Macfarlane et al., (2001) and the McBeth et al., (2009) likely due to the sample being restricted to adults aged 50 years and over.

\section{Methodological considerations}

\section{Sample structure}

It was not possible to test all of the pain phenotypes within the same dataset. Differences in the structure of the study population may have influenced the observed relationships between pain phenotype and mortality. ELSA samples are generated with the intention of being representative of the English population of adults aged 50 years and over. In contrast the NorStOP dataset is sampled from a small number $(n=8)$ of general practices in North Staffordshire and is less likely to be nationally representative. However, the age and sex structure of the participating NorStOP population is similar to that of North Staffordshire and to England and Wales (28). Investigation of the age and sex distribution of the samples used for this study revealed that the ELSA and NorStOP samples had similar structures to 
each other. Although selective samples were used and some differences in demographic and socioeconomic characteristics between those with and without missing data were indicated, there was sufficient power to detect the expected effect sizes (based on previous literature) and a weighted analysis indicated the missing data did not introduce bias to the results.

Identification of vital status

Vital status in the ELSA sample was determined using records from the Office of National Statistics whereas in NorStOP information was collated from two sources, the Exeter Patient registration system held at the local Primary Care Trust and through manual tracing of the NHS Summary Care Record Demographic system. Misclassifications may have occurred resulting in an under or over estimation of the true relationship between pain and mortality. However, the United Kingdom is considered to have high quality death registration data (29) so any inaccuracies should be minimal.

\section{Mortality information}

Only year of death information was available from the ELSA dataset for this study. This has implications for the accuracy of the observed MRRs. For participants who died, the time in the study was calculated from their date of interview until $31^{\text {st }}$ December in the year the participant was known to have died. The actual date of death may have been earlier meaning survival time was overestimated and mortality risk was underestimated for ELSA participants in the current study.

\section{Pain phenotype}


The current study used data obtained from two large population-based surveys with high response rates and it was possible to investigate the relationship between a number of different pain phenotypes and mortality. However, it was not possible to determine pain that was chronic (i.e. lasting for three months or more) in either dataset but this is unlikely to have affected the results as $80-90 \%$ of widespread pain is chronic (4). Pain was measured at one point in time only and it is possible participants may have been misclassified or changed pain state over the follow-up period. This would result in an inaccurate representation of the relationship between pain and mortality (either an under or overestimation) but the number of misclassifications of pain state is likely to be small, resulting in minimal effect on the overall findings.

Bias

It is possible that early deaths resulted from causes (such as cancer) that pre-dated the onset of pain, and this might have dominated our findings. Our analysis of pain interference, because it separated mortality in the first year of follow-up from later deaths (tables 4 and 5), provided an opportunity to examine this possibility. Although the point estimate of mortality risk was highest in the 'extreme interference' group in the first year, elevated risks of mortality after 12 months were seen for all grades of interference and were statistically more stable than those for year 1 . This finding argues against any substantial influence of this form of bias.

\section{Implications for further research}

The findings from the current study demonstrated an increased risk of mortality for people who had pain that impacted on their life. A measure of pain impact along with measures of 
presence and location would be a useful addition to any future studies of pain and mortality. One of the research implications that follows from our study is the need to identify what it is about 'interfering' pain that causes premature mortality. Factors such as comorbid disease, lifestyle behaviours or psychological factors might provide links between pain that specifically disables or interferes with life and premature mortality. For example, pain that impacts on life is linked to a reduction in physical activity which is associated with increasing weight and the onset of health conditions (e.g. cardiovascular disease) that lead to mortality. Future analyses could usefully explore such pathways to identify targets for intervention to reduce the impact of pain on daily life and hence on premature mortality.

\section{Conclusion}

The impact of pain was more important than the presence or extent of pain in the relationship between pain and mortality.

\section{Acknowledgements}

ELSA data were made available through the UK Data Archive (UKDA). ELSA was developed by a team of researchers based at the NatCen Social Research, University College London and the Institute for Fiscal Studies. The data were collected by NatCen Social Research. The funding is provided by the National Institute of Aging in the United States, and a consortium of UK government departments co-ordinated by the Office for National Statistics. The developers and funders of ELSA and the Archive do not bear any responsibility for the analyses or interpretations presented here.

There are no conflicts of interest. 


\section{References}

1. Woolf AD, Erwin J, March L. The need to address the burden of musculoskeletal conditions. Best Pract Res Clin Rheumatol 2012;26:183-224. Available at:

http://linkinghub.elsevier.com/retrieve/pii/S1521694212000368. Accessed July 16, 2012.

2. Woolf AD, Pfleger B. Burden of major musculoskeletal conditions. Bull World Health Organ 2003;81:646-656. Available at:

http://www.scielosp.org/scielo.php?script=sci_arttext\&pid=S0042-96862003000900007. Accessed August 29, 2012.

3. Murray CJL, Vos T, Lozano R, Naghavi M, Flaxman AD, Michaud C, et al. Disability-adjusted life years (DALYs) for 291 diseases and injuries in 21 regions, 1990-2010: a systematic analysis for the Global Burden of Disease Study 2010. Lancet 2012;380:2197-223. Available at: http://www.ncbi.nlm.nih.gov/pubmed/23245608. Accessed July 10, 2014.

4. Macfarlane GJ, McBeth J, Silman AJ. Widespread body pain and mortality: prospective population based study. Bmj 2001;323:1-5.

5. Macfarlane GJ, Jones GT, Knekt P, Aromaa a, McBeth J, Mikkelsson M, et al. Is the report of widespread body pain associated with long-term increased mortality? Data from the Mini-Finland Health Survey. Rheumatology (Oxford) 2007;46:805-7. Available at: http://www.ncbi.nlm.nih.gov/pubmed/17189245. Accessed August 29, 2012.

6. Andersson $\mathrm{HI}$. Increased mortality among individuals with chronic widespread pain relates to lifestyle factors: a prospective population-based study. Disabil Rehabil 2009;31:1980-7. Available at: http://www.ncbi.nlm.nih.gov/pubmed/19874076. Accessed August 20, 2012.

7. McBeth J, Symmons DP, Silman a J, Allison T, Webb R, Brammah T, et al. Musculoskeletal pain is associated with a long-term increased risk of cancer and cardiovascular-related mortality. Rheumatology (Oxford) 2009;48:74-7. Available at:

http://www.pubmedcentral.nih.gov/articlerender.fcgi?artid=2639482\&tool=pmcentrez\&ren dertype=abstract. Accessed August 29, 2012.

8. Torrance N, Elliott A. Severe chronic pain is associated with increased 10 year mortality. A cohort record linkage study. Eur J Pain 2010;14:380-386. Available at:

http://onlinelibrary.wiley.com/doi/10.1016/j.ejpain.2009.07.006/full. Accessed August 29, 2012.

9. Nitter $A K$, Forseth $K \varnothing$. Mortality rate and causes of death in women with self-reported musculoskeletal pain: Results from a 17-year follow-up study. Scand J Pain 2013;4:86-92. Available at: http://linkinghub.elsevier.com/retrieve/pii/S1877886012002443. Accessed May 8, 2013.

10. Dreyer L, Kendall S, Danneskiold-Samsøe B, Bartels EM, Bliddal H. Mortality in a cohort of Danish patients with fibromyalgia: increased frequency of suicide. Arthritis Rheum 2010;62:3101-8. Available at: http://www.ncbi.nlm.nih.gov/pubmed/20583101. Accessed August 2, 2012.

11. Wolfe F, Hassett AL, Walitt B, Michaud K. Mortality in fibromyalgia: a study of 8,186 
patients over thirty-five years. Arthritis Care Res (Hoboken) 2011;63:94-101. Available at: http://www.ncbi.nlm.nih.gov/pubmed/20662040. Accessed August 29, 2012.

12. Sjøgren P, Grønbæk M. A population-based cohort study on chronic pain: the role of opioids. Clin J Pain 2010;26:763-769. Available at:

http://journals.Iww.com/clinicalpain/Abstract/2010/11000/A_Population_based_Cohort_St udy_on_Chronic_Pain_.5.aspx. Accessed March 29, 2014.

13. Smith BH, Elliott AM, Hannaford PC. Pain and subsequent mortality and cancer among women in the Royal College of General Practitioners Oral Contraception Study. Br J Gen Pract 2003;53:45-6. Available at:

http://www.pubmedcentral.nih.gov/articlerender.fcgi?artid=1314492\&tool=pmcentrez\&ren dertype=abstract.

14. Smith D, Wilkie R, Uthman O, Jordan JL, McBeth J. Chronic pain and mortality: a systematic review. PLoS One 2014;9:e99048. Available at:

http://www.pubmedcentral.nih.gov/articlerender.fcgi?artid=4047043\&tool=pmcentrez\&ren dertype=abstract. Accessed March 6, 2015.

15. Tang NKY, Crane C. Suicidality in chronic pain: a review of the prevalence, risk factors and psychological links. Psychol Med 2006;36:575-86. Available at:

http://www.ncbi.nlm.nih.gov/pubmed/16420727. Accessed August 2, 2012.

16. Wolfe F, Clauw DJ, Fitzcharles M-A, Goldenberg DL, Katz RS, Mease P, et al. The American College of Rheumatology preliminary diagnostic criteria for fibromyalgia and measurement of symptom severity. Arthritis Care Res (Hoboken) 2010;62:600-10. Available at: http://www.ncbi.nlm.nih.gov/pubmed/20461783. Accessed July 12, 2012.

17. Marmot, M., Oldfield, Z., Clemens, S., Blake, M., Phelps, A., Nazroo, J., Steptoe, A., Rogers, N., Banks J. English Longitudinal Study of Ageing: Waves 0-5, 1998-2011. 19th Edition. Available at: http://www.ifs.org.uk/ELSA.

18. NatCen Social Research. English Longitudinal Study of Ageing (ELSA) Waves 1 to 6. User guide to the core datasets.; 2014.

19. Thomas E, Wilkie R, Peat G, Hill S, Dziedzic K, Croft P. The North Staffordshire Osteoarthritis Project--NorStOP: prospective, 3-year study of the epidemiology and management of clinical osteoarthritis in a general population of older adults. $B M C$ Musculoskelet Disord 2004;5:2.

20. Wolfe F, Smythe H, Yunus M, Bennett R, Bombardier C, Goldenberg DL, et al. The American College of Rheumatology 1990 criteria for the classification of fibromyalgia. Arthritis Rheum 1990;33:160-172. Available at:

http://onlinelibrary.wiley.com/doi/10.1002/art.1780330203/abstract. Accessed August 20, 2012.

21. Hunt IM, Silman a J, Benjamin S, McBeth J, Macfarlane GJ. The prevalence and associated features of chronic widespread pain in the community using the "Manchester" definition of chronic widespread pain. Rheumatology (Oxford) 1999;38:275-9. Available at: http://www.ncbi.nlm.nih.gov/pubmed/10325667. 
22. Ware JE, Kosinski KM, Bayliss MS. Comparison of methods for scoring and statistical analysis of the SF-36 health profile and summary measures: results from the medical outcomes study. Med Care 1995;33q:AS264-AS279.

23. Demakakos $P$, Nazroo J, Breeze E, Marmot M. Socioeconomic status and health: The role of subjective social status. Soc Sci Med 2008;67:330-340.

24. Litwin H, Sapir E V. Perceived income adequacy among older adults in 12 countries: Findings from the survey of health, ageing, and retirement in Europe. Gerontologist 2009;49:397-406.

25. Jordan K, Croft P. Mortality and cancer in patients with new musculoskeletal episodes: a cohort study. Br J Gen Pract 2010:105-112. Available at:

http://www.ncbi.nlm.nih.gov/pmc/articles/PMC2828857/. Accessed August 29, 2012.

26. Kamaleri Y, Natvig B, Ihlebaek CM, Bruusgaard D. Localized or widespread musculoskeletal pain: does it matter? Pain 2008;138:41-6. Available at: http://www.ncbi.nlm.nih.gov/pubmed/18077092. Accessed August 20, 2012.

27. Lacey RJ, Lewis M, Jordan K, Jinks C, Sim J. Interrater reliability of scoring of pain drawings in a self-report health survey. Spine (Phila Pa 1976) 2005;30:E455-E458.

28. Thomas E, Mottram S, Peat G, Wilkie R, Croft P. The effect of age on the onset of pain interference in a general population of older adults: prospective findings from the North Staffordshire Osteoarthritis Project (NorStOP). Pain 2007;129:21-7. Available at: http://www.ncbi.nlm.nih.gov/pubmed/17084980. Accessed September 1, 2012.

29. Mathers CD, Fat DM, Inoue M, Rao C, Lopez AD. Counting the dead and what they died from: An assessment of the global status of cause of death data. Bull World Health Organ 2005;83:171-177. 
Table 1. Cross tabulation of the number of pain sites and pain interference categories presenting the proportion of those who died in the NorStOP sample $(n=10953)$ * $\%$ who died (Number of deaths/N) per cell

\begin{tabular}{|c|c|c|c|c|c|c|}
\hline & No sites & $1-3$ sites & 4-6 sites & 7-11 sites & $12+$ sites & Total \\
\hline No & $13.0 \%$ & $6.7 \%$ & $6.9 \%$ & $6.2 \%$ & $4.0 \%$ & $11.1 \%$ \\
\hline interference & $(412 / 3166)$ & $(46 / 683)$ & $(26 / 376)$ & $(12 / 195)$ & $(3 / 75)$ & (499/4495) \\
\hline \multirow[t]{2}{*}{ A little } & - & $11.3 \%$ & $10.1 \%$ & $8.5 \%$ & $7.9 \%$ & $9.8 \%$ \\
\hline & & $(81 / 714)$ & $(72 / 716)$ & $(48 / 565)$ & $(25 / 316)$ & $(226 / 2311)$ \\
\hline \multirow[t]{2}{*}{ Moderately } & - & $19.8 \%$ & $17.2 \%$ & $13.0 \%$ & $11.4 \%$ & $15.0 \%$ \\
\hline & & $(59 / 298)$ & (71/414) & $(57 / 440)$ & $(46 / 404)$ & $(233 / 1556)$ \\
\hline \multirow[t]{2}{*}{ Quite a bit } & - & $20.9 \%$ & $24.5 \%$ & $17.5 \%$ & $15.9 \%$ & $18.4 \%$ \\
\hline & & $(47 / 225)$ & $(87 / 355)$ & $(89 / 510)$ & $(144 / 904)$ & (367/1994) \\
\hline \multirow[t]{2}{*}{ Extremely } & - & $37.5 \%$ & $24.7 \%$ & $28.5 \%$ & $23.8 \%$ & $25.6 \%$ \\
\hline & & $(12 / 32)$ & $(20 / 81)$ & $(35 / 123)$ & $(86 / 361)$ & $(153 / 597)$ \\
\hline \multirow[t]{2}{*}{ Total } & $13.0 \%$ & $12.6 \%$ & $14.2 \%$ & $13.2 \%$ & $14.8 \%$ & $13.5 \%$ \\
\hline & $(412 / 3166)$ & $(245 / 1952)$ & $(276 / 1942)$ & (241/1833) & (304/2060 & (1478/10953) \\
\hline
\end{tabular}

*excludes $\mathrm{n}=32$ who indicated the presence of pain but did not indicate any pain sites on the pain manikin 
Table 2. Risk of all-cause mortality in the ELSA sample according to pain phenotype $(n=6324)$ and weighted results

\begin{tabular}{|c|c|c|c|c|c|c|c|c|c|c|}
\hline Pain & $\mathbf{N}$ & Person years & Number & Mortality & Model 1 & Model 2 & Model 3 & Weighted & Weighted & Weighted \\
\hline \multirow[t]{3}{*}{ phenotype } & & of follow up & of deaths & rate* & MRR (95\% & MRR $(95 \%$ & MRR (95\% & Model 1 & Model 2 & Model 3 \\
\hline & & & & & \multirow[t]{2}{*}{ CI) } & \multirow[t]{2}{*}{ Cl) } & \multirow[t]{2}{*}{ Cl) } & MRR & MRR & MRR \\
\hline & & & & & & & & $(95 \% \mathrm{Cl})$ & $(95 \% \mathrm{Cl})$ & $(95 \% \mathrm{Cl})$ \\
\hline Not often & 4052 & 28048 & 428 & 15.26 & Reference & Reference & Reference & Reference & Reference & Reference \\
\hline \multicolumn{11}{|l|}{ troubled } \\
\hline Often & 2272 & 15414 & 336 & 21.80 & 1.43 (1.24, & 1.36 (1.18, & 1.29 (1.12, & 1.43 (1.24, & 1.37 (1.18, & 1.29 (1.11, \\
\hline troubled & & & & & 1.65) & 1.58) & 1.49) & 1.65) & 1.58) & 1.50) \\
\hline Not often & 4052 & 28048 & 428 & 15.26 & Reference & Reference & Reference & Reference & Reference & Reference \\
\hline \multicolumn{11}{|l|}{ troubled } \\
\hline \multirow[t]{2}{*}{ Mild } & 680 & 4737 & 60 & 12.67 & $0.83(0.63$ & $0.87(0.66$ & $0.89(0.68$ & $0.82(0.63$ & 0.85 (0.65, & 0.87 (0.67, \\
\hline & & & & & 1.09) & 1.14) & 1.16) & 1.08) & 1.12) & 1.14) \\
\hline \multirow[t]{2}{*}{ Moderate } & 1183 & 7943 & 201 & 25.31 & 1.65 (1.40, & 1.52 (1.28, & 1.42 (1.20, & 1.68 (1.42, & 1.54 (1.30, & 1.43 (1.20, \\
\hline & & & & & 1.95) & $1.80)$ & 1.68) & 1.98) & 1.84) & $1.72)$ \\
\hline \multirow[t]{2}{*}{ Severe } & 409 & 2734 & 75 & 27.43 & 1.81 (1.41, & $1.70(1.33$ & 1.54 (1.20, & 1.80 (1.41, & 1.70 (1.31, & 1.53 (1.19, \\
\hline & & & & & 2.31) & 2.18) & 1.97) & 2.31) & 2.19) & 1.98) \\
\hline
\end{tabular}

Model 1: Crude

Model 2: Adjusted for age, sex

Model 3: Adjusted for age, sex, education and wealth

*per 1000 person-years

MRRs in bold indicate significant associations 
Table 3. Risk of all-cause mortality in the NorStOP sample according to pain phenotype ( $n=10985)$ and weighted results

\begin{tabular}{|c|c|c|c|c|c|c|c|c|c|c|}
\hline Pain & $\mathbf{N}$ & Person & Number & Mortality & Model 1 & Model 2 & Model 3 & Weighted & Weighted & Weighted \\
\hline \multirow[t]{3}{*}{ phenotype } & & years of & of deaths & rate* & MRR & MRR & MRR & Model 1 & Model 2 & Model 3 \\
\hline & & follow up & & & $(95 \% \mathrm{Cl})$ & $(95 \% \mathrm{Cl})$ & $(95 \% \mathrm{Cl})$ & MRR & MRR & MRR \\
\hline & & & & & & & & $(95 \% \mathrm{Cl})$ & (95\% Cl) & (95\% Cl) \\
\hline No pain & 3166 & 28813 & 412 & 14.30 & Reference & Reference & Reference & Reference & Reference & Reference \\
\hline \multirow[t]{2}{*}{ Any pain } & 7819 & 71116 & 1072 & 15.07 & 1.05 (0.94, & $1.08(0.96$, & 1.06 (0.95, & 1.00 (0.89, & 1.08 (0.96, & 1.06 (0.95, \\
\hline & & & & & 1.18) & 1.21) & 1.19) & 1.12) & $1.20)$ & 1.19) \\
\hline No pain & 3166 & 28813 & 412 & 14.30 & Reference & Reference & Reference & Reference & Reference & Reference \\
\hline Pain but not & 5038 & 45709 & 714 & 15.62 & 1.09 (0.97, & 1.06 (0.94, & 1.05 (0.93, & 1.06 (0.94, & 1.07 (0.95, & 1.06 (0.94, \\
\hline ACR WP & & & & & 1.23) & 1.20) & 1.19) & 1.19) & 1.20) & 1.19) \\
\hline \multirow[t]{2}{*}{ ACR WP } & 2749 & 25158 & 352 & 13.99 & $0.97(0.85$ & $1.10(0.95$ & 1.07 (0.92, & $0.90(0.78$ & 1.09 (0.95, & $1.07(0.92$, \\
\hline & & & & & 1.12) & 1.26) & 1.23) & 1.04) & 1.26) & 1.23) \\
\hline No pain & 3166 & 28813 & 412 & 14.30 & Reference & Reference & Reference & Reference & Reference & Reference \\
\hline Pain but not & 6062 & 55194 & 827 & 14.98 & $1.05(0.93$ & 1.05 (0.93, & 1.03 (0.92, & 1.02 (0.90, & 1.05 (0.93, & $1.04(0.92$ \\
\hline Manchester & & & & & 1.18) & 1.18) & 1.16) & 1.14) & 1.18) & 1.17) \\
\hline \multicolumn{11}{|l|}{ WP } \\
\hline Manchester & 1725 & 15673 & 239 & 15.25 & 1.06 (0.91, & 1.19 (1.02, & 1.16 (0.99, & 0.94 (0.80, & 1.19 (1.01, & 1.16 (0.99, \\
\hline WP & & & & & $1.25)$ & 1.40) & $1.36)$ & 1.11) & 1.39) & $1.36)$ \\
\hline No sites & 3166 & 28813 & 412 & 14.30 & Reference & Reference & Reference & Reference & Reference & Reference \\
\hline \multirow[t]{2}{*}{ 1-3 sites } & 1952 & 17843 & 245 & 13.73 & $0.96(0.82$ & 0.95 (0.81, & 0.95 (0.81, & 0.95 (0.81, & 0.94 (0.81, & 0.94 (0.80, \\
\hline & & & & & 1.12) & 1.11) & 1.11) & 1.11) & 1.10) & 1.10) \\
\hline \multirow[t]{2}{*}{ 4-6 sites } & 1942 & 17559 & 276 & 15.72 & 1.10 (0.94, & 1.09 (0.94, & 1.08 (0.93, & 1.07 (0.92, & 1.09 (0.93, & 1.07 (0.92, \\
\hline & & & & & $1.28)$ & 1.27) & 1.26) & 1.24) & 1.26) & $1.25)$ \\
\hline \multirow[t]{2}{*}{$7-11$ sites } & 1833 & 16836 & 241 & 14.31 & $1.00(0.85$ & 1.07 (0.92, & 1.05 (0.90, & $0.94(0.80$ & 1.06 (0.91, & 1.05 (0.89, \\
\hline & & & & & 1.17) & 1.26) & 1.24) & 1.10) & $1.25)$ & 1.23) \\
\hline \multirow[t]{2}{*}{$12+$ sites } & 2060 & 18630 & 304 & 16.32 & 1.14 (0.98, & $1.18(1.02$ & 1.15 (0.99, & 1.01 (0.87, & 1.17 (1.01, & 1.14 (0.99, \\
\hline & & & & & $1.32)$ & 1.37) & 1.34) & 1.17) & 1.36) & 1.33) \\
\hline
\end{tabular}

MRR= Mortality Rate Ratio, 95\% Cl = 95\% confidence interval, ACR = American College of Rheumatology, WP = Widespread pain

Model 1: Crude, Model 2: Adjusted for age, sex, Model 3: Adjusted for age, sex, education, adequacy of income

*per 1000 person-years, MRRs in bold indicate significant associations 
Table 4. Risk of all-cause mortality in the NorStOP sample according to pain interference in the first year of follow up (0-365 days)

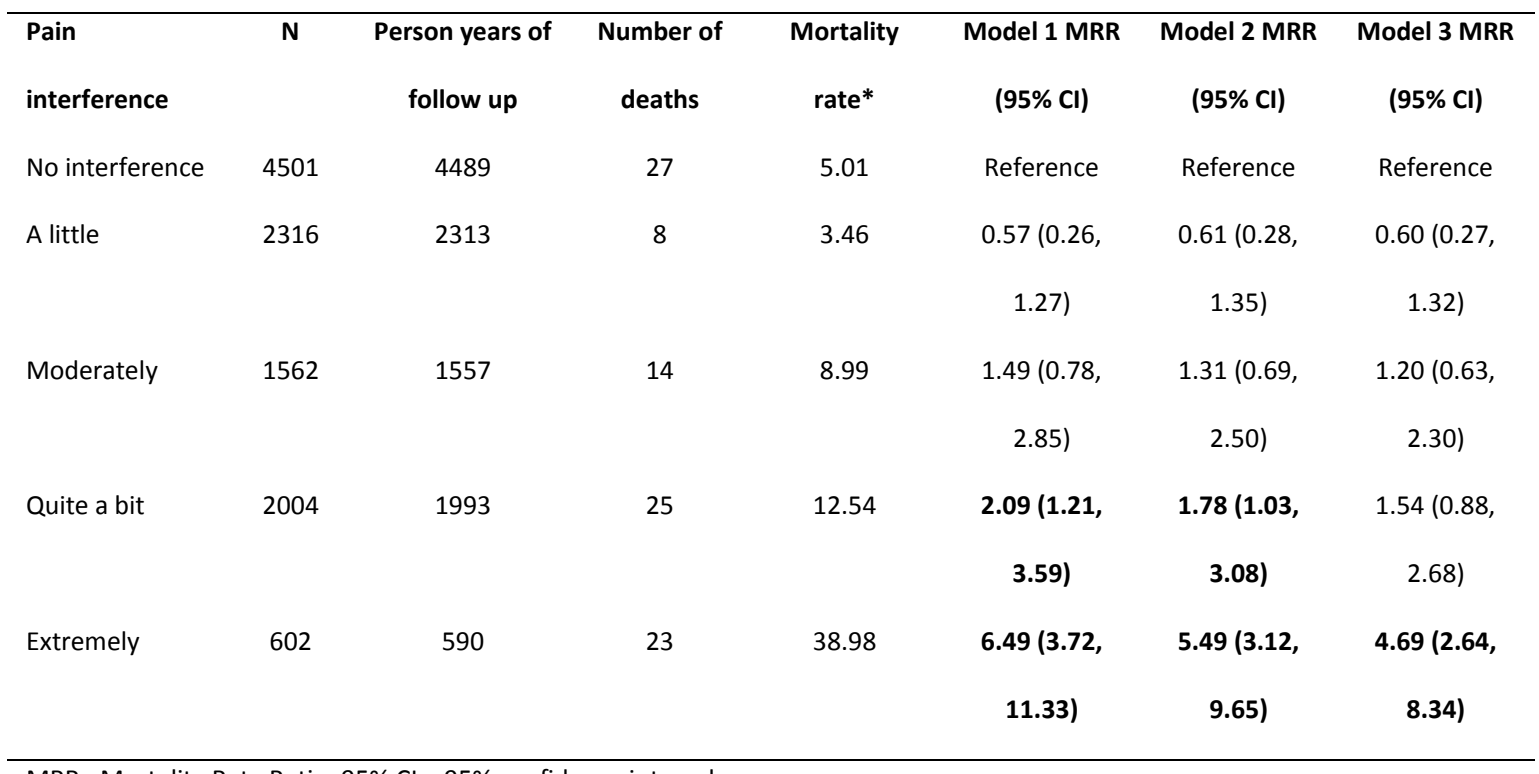

MRR= Mortality Rate Ratio, 95\% $\mathrm{Cl}=95 \%$ confidence interval

Model 1: Unadjusted

Model 2: Adjusted for age, sex

Model 3: Adjusted for age, sex, education, adequacy of income

*per 1000 person-years

MRRs in bold indicate significant associations 
Table 5. Risk of all-cause mortality in the NorStOP sample according to pain interference after the first year of follow up (365 to 3483 days)

\begin{tabular}{|c|c|c|c|c|c|c|c|}
\hline Pain & $\mathbf{N}$ & Person & Number & Mortality & Model 1 & Model 2 & Model 3 \\
\hline \multirow[t]{2}{*}{ interference } & & years of & of deaths & rate* & MRR (95\% & MRR (95\% & MRR $195 \%$ \\
\hline & & follow up & & & $\mathrm{CI})$ & $\mathrm{Cl})$ & $\mathrm{Cl})$ \\
\hline No & 4474 & 41269 & 472 & 11.44 & Reference & Reference & Reference \\
\hline \multicolumn{8}{|l|}{ interference } \\
\hline \multirow[t]{2}{*}{ A little } & 2308 & 21473 & 219 & 10.20 & 0.89 (0.76, & 0.94 (0.80, & $0.94(0.80$, \\
\hline & & & & & 1.04) & 1.10) & 1.10) \\
\hline \multirow[t]{2}{*}{ Moderately } & 1548 & 14238 & 220 & 15.45 & 1.35 (1.15, & $1.13(0.96$ & $1.13(0.96$, \\
\hline & & & & & 1.58) & 1.33) & 1.32) \\
\hline \multirow[t]{2}{*}{ Quite a bit } & 1979 & 17817 & 345 & 19.36 & 1.70 (1.48, & 1.39 (1.21, & 1.38 (1.20, \\
\hline & & & & & 1.95) & $1.60)$ & 1.59) \\
\hline \multirow[t]{2}{*}{ Extremely } & 579 & 5078 & 131 & 25.80 & 2.27 (1.87, & 1.89 (1.55, & 1.88 (1.54, \\
\hline & & & & & 2.76) & 2.30) & 2.29) \\
\hline
\end{tabular}

MRR= Mortality Rate Ratio, $95 \% \mathrm{Cl}=95 \%$ confidence interval

Model 1: Unadjusted

Model 2: Adjusted for age, sex

Model 3: Adjusted for age, sex, education, adequacy of income

* per 1000 person-years

MRRs in bold indicate significant associations 